\title{
0 acesso de primeiro contato como parâmetro da resolutividade na atenção primária
}

\author{
First-contact access as a parameter of resolutivity in primary care
}

Rita Oliveira Costa

Maitê da Veiga Feitoza Borges SILVA II

Carolina Freitas do Carmo

RODRIGUES

Ruhena Kelber Abrão Ferreira ${ }^{\mathrm{IV}}$

Cristina Vianna Moreira dos

SANTOS $^{\mathrm{IV}}$

'Fundação Escola De Saúde Pública

De Palmas (FESP), Palmas/TO -

Brasil

"Capitalent Medical, Alemanha

IIIInstituto Brasileiro De Desenvolvimento Da Administração Hospitalar (IBDAH), Salvador/BA - Brasil

${ }^{\text {IV } U n i v e r s i d a d e ~ F e d e r a l ~ D o ~ T o c a n t i n s ~}$ (UFT), Palmas/TO - Brasil
Resumo - Introdução: Este trabalho refere-se a um estudo teórico e descritivo com abordagem avaliativa, construído com base na leitura do manual do Programa de Melhoria do Acesso e Qualidade na Atenção Básica (PMAQ-AB), e na necessidade de discutir entraves da atenção primária no caráter resolutivo da mesma. Objetivo: Avaliar a capacidade resolutiva da atenção primária em saúde, tendo como parâmetro o atributo "acesso de primeiro contato". Método: Pesquisa exploratória em documentos no formato eletrônico, nas principais bases de dados da Biblioteca Virtual da Saúde (BVS), com a utilização de descritores em Ciências da Saúde. Foram utilizados 13 trabalhos relevantes, disponibilizados na íntegra, publicados nos últimos doze anos. Resultados: As pesquisas demonstraram que o atributo acesso de primeiro contato obteve conceitos insatisfatórios em todas as avaliações, estando associado aos serviços de referência indiscriminada, pouco vínculo, integralidade prejudicada e baixa resolutividade no cuidado primário. Conclusão: Dessa forma, expõe-se a necessidade urgente de reorganização do serviço com foco na integralidade e no cuidado continuado.

Palavras-chave: Atenção primária À saúde; Avaliação da QuaLIDAdE DA AssistÊnCIA À SAÚdE; Política de SAÚdE.

ABSTRACT - Introduction: This work refers to a theoretical and descriptive study with an evaluative approach, built based on reading the manual of the Program for Improvement of Access and Quality in Primary Care (PMAQ), and the need to discuss obstacles of primary care in the character resolutive of the same. Objective: To evaluate the resolution capacity of primary health care, using the attribute "first contact access" as a parameter. Method: Exploratory search of documents in electronic format, in the main databases of the Virtual Health Library (VHL), using descriptors in Health Sciences. 13 relevant works were used, made available in full published in the last twelve years. Results: Research has shown that the attribute of first contact access obtained unsatisfactory concepts in all assessments, being associated with indiscriminate reference services, little connection, impaired integrality, and low resolution in primary care. Conclusion: Thus, there is an urgent need to reorganize the service with a focus on integrality and continuous care.

Keywords: Primary health Care; Health Care Quality AssessMENT; HeALTH POLICY. 


\section{INTRODUÇÃO}

A Atenção Primária à Saúde (APS) pode ser interpretada, principalmente, de três maneiras: como atenção primária seletiva, como o nível primário do sistema de atenção à saúde e como estratégia de organização do sistema de atenção à saúde. Como atenção primária seletiva, Mendes ${ }^{1}$ a caracteriza como um:

programa específico destinado a populações e regiões pobres a quem se oferece, exclusivamente, um conjunto de tecnologias simples e de baixo custo, provido por pessoal de baixa qualificação profissional e sem a possibilidade de referência a níveis de atenção de maior densidade tecnológica (MENDES, 2015, p. 32).

Enquanto nível primário do sistema de atenção à saúde consiste em:

modo de organizar e fazer funcionar a porta de entrada do sistema, enfatizando a função resolutiva desses serviços sobre os problemas mais comuns de saúde, para o que a orienta de forma a minimizar os custos econômicos e a satisfazer as demandas da população, restritas, porém, às ações de atenção de primeiro nível. (MENDES, 2015, p. 32).

Já como estratégia de organização do sistema de atenção à saúde, sendo Mendes ${ }^{1}$ "uma forma singular de apropriar, recombinar e reordenar todos os recursos do sistema para satisfazer às necessidades, demandas e representações da população, o que implica a articulação da APS como parte e como coordenadora de uma RAS.

A conferência de Alma-Ata definiu a APS como:

cuidados essenciais baseados em métodos de trabalho e tecnologias de natureza prática, cien- tificamente críveis e socialmente aceitáveis, universalmente acessíveis na comunidade aos indivíduos e às famílias, com a sua total participação e a um custo suportável para as comunidades e para os países, à medida que se desenvolvem num espírito de autonomia e autodeterminação. (MENDES, 2015, p. 32).

A partir de 1978, sob os atos da Conferência de Alma-Ata, iniciaram-se profundas reflexões sobre a potência e impacto referente a APS, embora ainda o sistema não tivesse sido desenvolvido de forma grandiosa, por meios das ponderações, definidas na Conferência. $^{2}$

No ano de 1994, na idealização da reorganização, construção e expansão da APS, criou-se a Estratégia Saúde da Família (ESF). ${ }^{3}$ Sob a definição da ESF, Brasil retrata-se que:

A ESF visa à reorganização da atenção básica
no País, de acordo com os preceitos do Sistema
Único de Saúde (SUS), e é tida como estraté-
gia de expansão, qualificação e consolidação da
atenção básica por favorecer uma reorientação
do processo de trabalho com maior potencial de
aprofundar os princípios, diretrizes e fundamen-
tos da atenção básica, de ampliar a resolutivida-
de e impacto na situação de saúde das pessoas e
coletividades, além de propiciar uma importante
relação custo-efetividade. (BRASIL, 2012).

A garantia dos serviços de saúde exposta na Constituição Federal de $1988^{4}$ prevê em seu artigo 198 (s.p.), que "as ações e serviços públicos de saúde devam integrar-se a uma rede regionalizada e hierarquizada e constituir-se a um sistema único e organizado, por meio da descentralização, atendimento integral e da participação comunitária”. A descentralização entendida como princípio, parte do pressuposto de: 
redistribuir poder e responsabilidade entre os três níveis de governo, de modo que a responsabilidade pela saúde deve ser descentralizada até o município, ou seja, devem ser fornecidas ao município condições gerenciais, técnicas, administrativas e financeiras para exercer esta função (BRASIL, 2017).

De uma forma simplificada, a gestão municipal tem a responsabilidade de garantir o acesso ao sistema de saúde por meio da APS. Para isso, acredita-se que o planejamento estratégico, a partir da promoção de mudanças no processo de trabalho, possa mitigar as barreiras de acesso à "porta de entrada"6, principalmente no que tange ao primeiro contato e manutenção do vínculo. Destaca-se que o conceito de "porta de entrada" do SUS foi atribuído à APS a partir da expansão da ESF.?

Para um problema de saúde, é necessário que esse ponto de entrada seja de fácil acesso e inerente à organização de serviços de saúde. ${ }^{7}$ Assim, também o Ministério da Saúde (MS) define, em conceitos complementares, o acesso de primeiro contato como sendo "a acessibilidade e utilização do serviço de saúde como fonte de cuidado a cada novo problema ou novo episódio de um mesmo problema de saúde" ${ }^{8}$. Em um sentido mais técnico, Azevedo e Costa ${ }^{9}$ retratam que a experiência de acesso dos indivíduos no contato direto ou indireto com os serviços de saúde, diz muito a respeito da capacidade do sistema em corresponder às suas expectativas e necessidades.

$\mathrm{O}$ atributo acesso de primeiro contato é quando a equipe e a população reconhecem o serviço como o primeiro recurso a ser procurado quando há uma necessidade de saúde, devendo ${ }^{10}$, assim, refletir a respeito das múltiplas singularidades dos milhões de cidadãos dependentes exclusivamente do SUS faz-se impresindível. Segundo a Pesquisa Nacional de Saúde ${ }^{11}$, a população brasileira possui uma forte dependência do SUS, visto que $71,5 \%$ das pessoas não tem acesso à saúde suplementar.

Partindo desse pressuposto, a garantia do acesso facilitado aos serviços de saúde infelizmente não garante a efetividade do cuidado em saúde, porém a ESF, enquanto proximidade com o território e determinantes do ususário, é o nível de atenção com maior potendoal para ampliar a resolutividade e impactar na situação de saúde das pessoas e da coletividade ${ }^{11}$. Assim, a resolutividade do serviço prestado está aquém do simples primeiro contato, mas sobressai-se ao princípio da integralidade.

Segundo Costa et al. ${ }^{12}$, a integralidade preconiza que, além de atividades de promoção, prevenção e tratamentos, a forma como as práticas de cuidado são realizadas nos serviços de saúde também são fundamentais para o alcance da resolubilidade do atendimento. $\mathrm{O}$ conceito de resolutividade está associado à resolução final dos problemas trazidos pelos usuários ao serviço e à satisfação desses e dos profissionais. ${ }^{12}$

Porém, o estudo de Almeida, Fausto e Giovanella $^{13}$, que analisou ações empreendidas em quatro centros urbanos para fortalecer a ESF no Brasil, evidenciou que os municípios brasileiros apresentam baixa resolutividade. Uma prova desta fragilidade é que tem-se observado o aumento de internações hospitalares por condições sensíveis a APS que representam um conjunto de problemas de saúde que, com a ação e orientação correta no nível primário de 
saúde, diminuiria o risco destas internações, indicando sérios problemas de acesso ao sistema de saúde ou de seu desempenho ${ }^{14}$.

Visando contribuir para a melhoria dos serviços que contemplam a APS, o presente estudo buscou avaliar a capacidade resolutiva da atenção primária em saúde, tendo como parâmetro o atributo acesso de primeiro contato.

\section{MeTódo}

Trata-se de um estudo teórico e descritivo com abordagem avaliativa, construído com base na leitura crítica do manual do Programa de Melhoria do Acesso e Qualidade na Atenção Básica (PMAQ-AB) - $3^{\circ}$ Ciclo, do Departamento de Atenção Básica do Ministério da Saúde, e em estudos científicos atuais que referenciam a qualificação do acesso de primeiro contato e resolutividade na atenção primária.

A pesquisa descritiva visa o estabelecimento de relações entre variáveis, envolvendo o uso de técnicas, questionários e a observação sistemática. Desse modo, os fatos são observados, analisados e registrados sem a interferência do pesquisador. A elaboração deste artigo seguiu os pressupostos da revisão de literatura que "consiste em um apanhado geral sobre os principais documentos e trabalhos realizados a respeito do tema escolhido, abordados anteriormente por outros pesquisadores para a obtenção de dados para a pesquisa" 9 .

O caminho metodológico incluiu primeiramente o levantamento bibliográfico, por meio do qual realizou-se uma pesquisa exploratória em formato eletrônico nas principais base de dados na área da saúde da Biblioteca Virtual da Saúde (BVS), Medical Literature Analysis and Retrieval System Online (Medline), Literatura Latino-Americana e do Caribe em Ciências da Saúde (Lilacs), Scientific Electronic Library Online (Scielo), Google Acadêmico (Google Scholar). Foram utilizados também manuais e documentos do MS como fonte de informações.

Os descritores foram: "acesso de primeiro contato", "avaliação da atenção básica", "resolutividade na APS”, "PMAQ-AB”. A escolha dessas palavras-chave foi realizada com base na leitura de produções relacionadas ao tema, obtendo, assim, embasamento prévio. A busca ocorreu no segundo semestre de 2018 e foram encontrados 158 artigos nessa primeira etapa. A segunda etapa constituiu na seleção dos trabalhos com base nos critérios de inclusão preestabelecidos: abordarem a avaliação do acesso, possuir relevância temática, publicações nos últimos doze anos e estarem dentro do objetivo deste trabalho.

Por objetividade da pesquisa, a resolutividade da APS foi discutida, por meio da análise das dificuldades identificadas no mais simples dos processos dentro do sistema, ou seja, o primeiro acesso, bem como na leitura primorosa de manuais do MS. Posteriormente, as qualificações positivas ou negativas sobre o acesso e acessibilidade, verificou-se a relação entre falha no nível primário e objetividade resolutiva da atenção primária por meio de uma reflexão teórica.

Para interpretação e aplicação das análises sobre os resultados coletados foi considerado o embasamento técnico científico dos revisores dos estudos. 


\section{RESULTADOS E DISCUSSÃo}

A busca na BVS remeteu a 158 artigos. A verificação do tema e da leitura desses resumos levou à exclusão de 123 trabalhos (76 por não haverem relação com a temática do estudo e 47 estavam duplicados na base de dados). Após essa pré-seleção permaneceram 35 artigos a serem avaliados quanto as variáveis, com as quais foram rejeitados 22 artigos após a leitura integral, por não abordarem métodos e avaliação da resolutividade, tampouco avaliação com resultados acerca do acesso de primeiro contato. Não obstante 12 artigos e o manual do PMAQ-AB explicativo constituem a amostra final deste estudo, totalizando 13 produções, conforme tabela a seguir:

Tabela 1 - Resultados encontrados a partir da estratégia de busca

\begin{tabular}{c|c|c}
\hline Base de dados & Amostragem & Amostragem final \\
\hline Google Scholar & 78 & 6 \\
\hline LILACS & 9 & - \\
\hline MEDLINE & 6 & 6 \\
\hline SCIELO & 65 & 1 \\
\hline $\begin{array}{c}\text { Outras fontes } \\
\text { (MS) }\end{array}$ & 1 & $\mathbf{1 3}$ \\
\hline TOTAL & $\mathbf{1 5 9}$ & \\
\hline
\end{tabular}

Fonte: próprios autores.

É pertinente abordar os atores que fazem parte desse processo de construção equitativa do SUS, principalmente quando se trata da avaliação dos serviços que abrangem toda a população dependente desse sistema de saúde. Quando se fala em avaliar a percepção e experiência dos diferentes atores, é importante salientar a noção de que a avaliação multidimensional é um processo que resulta, muitas vezes, na identificação de conflitos e na busca de soluções. ${ }^{16}$ Baseado nessa afirmação, os atores podem optar por formas de mediação entre esses conflitos.

A partir da amostra foi observado que o processo de saúde estava mais presente nas discussões sobre avaliação do acesso, sendo que 7 estudos $(58,2 \%)$ levaram em conside- ração a opinião dos usuários, 9 (75\%) a dos profissionais e apenas $4(33,3 \%)$ de gestores públicos. Declarou-se como ponto forte, a análise comparativa das três perspectivas, a qual Reis et. al. ${ }^{17}$ reafirmam a relevância da pesquisa ampliada das estruturas e processos que permeiam a consolidação da APS como porta de entrada do SUS.

Quanto ao atributo acesso de primeiro contato, o mesmo foi abordado como tendo um Escore Insatisfatório (segundo medida e cálculos do instrumento utilizado - PCATool). Os motivos são diversos, entre os quais: a unidade de saúde não funciona nos dias de sábado e domingo, ou, até mesmo, durante a semana no período noturno. ${ }^{18,19,20} \mathrm{O}$ mesmo resultado foi ob- 
servado no estudo realizado por Chomatas et. al. ${ }^{21}$, como também foram relatados a inexistência de um telefone para contato na unidade. ${ }^{18,19}$ Esses dados coincidem com os estudos de outros autores. ${ }^{17,22}$

O modelo de APS no Brasil é divergente em algumas questões propostas pelo instrumento PCATool, validado no Brasil, no que tange ao horário e dias e funcionamento. Isso pode explicar, em parte, o baixo escore em estudos relatados.

Também foi questionado quanto ao atendimento no mesmo dia, e se a marcação de consulta é facilitada. Um estudo realizado por Lins et al. ${ }^{18}$ evidenciou que a partir da ampliação da ESF, instituída pela Política Nacional de Atenção Básica (PNAB), unidades com locais geográficos próximos da população ${ }^{9}$, criação de Agentes Comunitários de Saúde (ACS) e visitas domiciliares, entre outras melhorias, avançaram no quesito facilidade de marcação de consulta e atendimento no mesmo dia.

Em divergência ao estudo de Lins et al. ${ }^{18}$, Oliveira $^{23}$, encontrou dificuldades no que se refere às barreiras geográficas, ocasionando a maior limitação ao acesso, entrevistando idosos acima de 60 anos. Entretanto, foi evidenciado o acesso facilitado em consultas médicas, de enfermagem e odontológicas em relação às consultas de emergência, como também às programadas. Esse último pode estar associado às prioridades nas marcações, ainda mais se o idoso for portador de alguma doença de base.

$O$ acesso também foi apresentado negativamente relacionando-o às consultas programadas. ${ }^{9,24}$ Nesse caso, a oferta não é consentida a todos que necessitam, no mesmo dia. ${ }^{17,25-26} \mathrm{O}$ agendamento é realiza- do em uma semana, e o atendimento a essa demanda só seria possível na semana conseguinte. $\mathrm{O}$ atendimento em casos agudos só é possível mediante a presença de médico especialista no estabelecimento, caso contrário, o sujeito é encaminhado à rede de emergência. ${ }^{9,26}$

$\mathrm{O}$ acesso sem integralidade da atenção limita o caráter universalizante do sistema. ${ }^{27}$ Essa afirmação é verdadeira, dado que, uma vez que a ESF juntamente com seus profissionais, recebem atribuições emergenciais, as mesmas devem ser de caráter resolutivo como também integral. Não há como desmembrar as condutas emergenciais da APS para estabelecimentos de urgência e emergência.

Outro estudo também identificou dificuldades no acesso, o qual correspondia à marcação de consultas, mostrando-se como um obstáculo a utilização dos serviços. Nesse caso, os atendimentos por demanda espontânea só eram possíveis por meio de um encaminhamento pelo ACS e, muitas vezes, com horários específicos. ${ }^{17,28}$

Práticas pouco receptivas também são comentadas e vistas nos ambientes de recepção e acolhimento das unidades de saú$\mathrm{de}^{26}$, o que dificulta ainda mais o acesso, visto que, o primeiro profissional a ouvir a problemática dos indivíduos é, geralmente, quem está nos serviços administrativos, na porta de entrada e recepção, e que evidentemente não corresponde às expectativas de quem necessita de atendimento. Nesse contexto, enfatiza-se a necessidade de haver um atendimento humanizado por parte de toda a equipe, fazendo com que os pacientes se sintam mais respeitados e dignos, já que o objetivo principal da APS é oferecer um ser- 
viço de saúde afetivo à população, melhorando assim o seu estado. ${ }^{29,30}$

A humanização pode apresentar diversos significados e naturezas subjetivas no lidar de cada um nos diferentes interesses de natureza ética, moral, emocional, entre outros. A proposta também vem com uma política de conquista por representar um conjunto de princípios e diretrizes em todos os níveis de atenção, práticas e serviços de saúde. ${ }^{31}$ Como recursos de humanização, os tecnológicos, o acesso, o acolhimento e o vínculo representam uma relação estabelecida entre trabalhadores e usuários, para que as ações de saúde sejam mais acolhedoras, ágeis e resolutivas. Assim, a primeira ação para garantir a humanização do atendimento a ser realizada por toda a equipe, ao receber a pessoa como paciente, é prestar-lhe acolhimento. ${ }^{32}$

O serviço de saúde, em especial a APS, deve estar preparado para executar sua mais importante função: acolher sem distinção os usuários adscritos, porém, não negando atendimento aos que não pertencerem ao território; assegurar o acesso, a integralidade, o cuidado continuado, sustentando a ideia de que é a principal, senão única, porta de entrada para outros serviços de saúde. Ante essas apropriações, a resolutividade na atenção é ponto imprescindível na qualidade do cuidado continuado, para tanto a rede assistencial necessita estar organizada e alicerçada em dois pilares: integralidade e capacidade resolutiva. ${ }^{33}$

A integralidade pode ser compreendida como:

a integração das ações programáticas e demanda espontânea; articulando as ações de promoção à saúde, prevenção de agravos, vigilância à saúde, tratamento e reabilitação e manejo das diversas tecnologias de cuidado e de gestão necessárias a estes fins e à ampliação da autonomia dos usuários e coletividades; trabalhando de forma multiprofissional, interdisciplinar e em equipe; realizando a gestão do cuidado integral do usuário e coordenando-o no conjunto da rede de atenção. (BRASIL, p. 21).

A resolutividade é definida como o ato de solucionar, dar solução a algo. "Nos serviços de saúde, a resolutividade é uma forma de avaliar esses serviços por meio dos resultados obtidos durante o atendimento ofertado ao usuário" ${ }^{33}$ (34). Para avaliar a resolutividade dentro do primeiro nível de atenção foi utilizado como material o Manual do PMAQ-AB em seu $3^{\circ}$ ciclo de atuação.

O programa tem avaliado os serviços da APS por meio de eixos reguladores da qualidade e assistência prestada. Entre eles compõe-se o Eixo Resolutividade, ao qual é explicado como sendo calculado o percentual de encaminhamentos médicos para um serviço especializado em um período considerado, em relação a quantificação de atendimentos que esse profissional realiza no mês. $\mathrm{O}$ indicador permite verificar o nível de resolutividade na APS, a fim de identificar a necessidade de intervenções em áreas específicas, melhorando a organização da oferta do cuidado em saúde entre os pontos da rede de atenção à saúde. ${ }^{5}$

Como dito anteriormente, a ordenação do acesso e realização de encaminhamentos (com relevância dos casos prioritários e com maior risco) que necessitem de opiniões e cuidados especializados deve ser orientada pela unidade porta de entrada.

Azevedo e Costa $^{9}$ enfatizam em uma análise comparativa, que as maiores dificuldades relacionam-se ao número excessivo 
de encaminhamentos realizados pela APS, por entender que há casos solucionáveis no âmbito da unidade que, no entanto, são encaminhados. Em outra pesquisa, de cunho avaliativo, remete-se ao fato dos homens utilizarem mais a atenção especializada para questões sensíveis a atenção básica, o que acaba por sobrecarregar os setores de urgência e emergência ${ }^{17}$ com urgências verdes ou azuis. Tem-se considerado a rede de emergência a porta de entrada para o serviço de saúde em um estudo realizado por Oliveira et. al. ${ }^{26}$, quando o objetivo é o atendimento a crianças com episódios agudos de problemas de saúde.

Mendes ${ }^{1}$ enfatiza que não se é possível organizar as redes de urgência e emergência sem retirar dessas os atendimentos de menor prioridade, mas que, para tanto, é necessário e ainda mais urgente que esse nível de atenção (APS) esteja adequado para a resolução de tal problemática. Acrescenta-se ainda que "a desarticulação dos diferentes níveis de atenção do sistema de saúde confirma o conhecimento empírico da realidade, retratada historicamente pela supervalorização do enfoque curativo e hospitalocêntrico ${ }^{21}$ " (35, p. 2), exigindo, assim, maior resolutividade e acessibilidade à APS.

Soma-se ao exposto que se, por um lado, os encaminhamentos são referenciados de forma indiscriminada pela APS, os serviços de contrarreferência soam piores, quando associados a continuidade do cuidado ou fechamento de casos. ${ }^{12}$ Observando os resultados de seu estudo, César et. al..$^{22}$ descreveram que tanto as UBSs quanto as ESFs recebem menos da metade dos resultados de interconsultas, dificultando ainda mais seu papel integrador, no qual não se observa o acompanha- mento do paciente pela equipe, tampouco a longitudinalidade da assistência prestada, dificultando a integralidade do cuidado.

$\mathrm{O}$ uso indistinto de encaminhamentos é antagônico ao que propõe o PMAQ-AB. O manual faz referência à ampliação do cuidado, resolubilidade da APS, otimização de recursos e maior eficiência e equidade nas filas de esperas. Tanto que seu uso serve como subsídio para projetos de planejamento, gestão e avaliação de políticas públicas voltadas para a qualidade e resolutividade da APS. ${ }^{30}$

O parâmetro instituído para os encaminhamentos médicos para os serviços especializados ao mês é de $5 \%$ a $20 \%$ e o calculo é obtido a partir da seguinte fórmula

Número de encaminhamentos médicos para serviço especializado ${ }^{\star} 10$

Número total de atendimentos médicos individuais

Pois bem, quanto maior o valor do numerador, menor é a capacidade resolutiva da APS. Em estudos que avaliaram as internações por condições sensíveis à APS, a grande maioria estava associada à inexistência de equipes de saúde da família, dificuldades no acesso, dificuldades no sistema de referência e contrarreferência, falta de vínculo com a estratégia, características organizativas próprias da atenção primária, e corroboraram para o aumento em duas vezes maior da probabilidade de internações sensíveis ao cuidado primário. ${ }^{14,36-37}$ Isso remete que o nível primário não executa de forma oportuna e apropriada o acompanhamento de seus indivíduos, tampouco reconhece sua essência como mantenedora dos processos resolutivos em saúde.

Buscando soluções para as fragilidades apresentadas, destacamos a ampliação do 
catálogo de provas diagnósticas solicitadas diretamente pelo médico de família e a disponibilidade de medicamentos na USF, a garantia de acesso a medicamentos, as capacitações para desenvolvimento de habilidades ou competências técnicas em áreas específicas, a presença de especialistas, a realização de matriciamento. ${ }^{13,38-39}$ Porém não há consenso sobre qual seria a resolutividade esperada dos serviços de APS. Segundo Vázquez et $a l .{ }^{40}$, redes integradas de atenção em saúde caracterizam-se por forte função de gatekeeping pela APS, já que provavelmente a maior parte dos problemas de saúde pode ser resolvida nesse nível de atenção ${ }^{41}$.

Assim sendo, o MS por meio do manual PMAQ-AB propõe ações de melhoria do indicador de resolutividade, dentre elas: a capacitação/atualização contínua dos profissionais, o monitoramento e a avaliação da qualidade e consistência dos dados informados pelas equipes com vistas ao acompanhamento da evolução de resultados, a negociação/contratualização de metas, a definição de prioridades de apoio institucional, $\mathrm{o}$ assessoramento à gestão, o aumento da capacidade clínica das equipes, a qualificação dos encaminhamentos de usuários para a atenção especializada, a pactuação de fluxos e protocolos, bem como a incorporação das ferramentas de telessaúde articu- lada às decisões clínicas e aos processos de regulação do acesso. ${ }^{41}$

\section{CONSIDERAÇõeS FINAIS}

Diante do exposto, gestores e equipes de saúde, como também usuários, necessitam de uma compreensão maior sobre seu papel e influência que incidem na APS. É necessário que haja mudança nas práticas que vêm ocorrendo dentro da saúde pública, robustecendo a assistência e colaborando para a autonomia dos serviços primários.

Em muitos dos estudos que foram detalhados neste trabalho, a maior parte deles identificaram que as unidades de saúde não atuam como reguladoras do sistema de saúde, não promovem ações programáticas e utilizam dos serviços de referência como meio de escape as suas próprias obrigações. Quando as suas estruturas são comparadas aos critérios que permeiam a resolutividade do nível primário por meio do PMAQ-AB, as unidades não alcançam os resultados esperados, no que tange a avaliação teórica.

Por fim, acredita-se que é fundamental que o nível primário utilize de uma metodologia voltada ao acolhimento a demanda espontânea que funcione com necessidade urgente de melhora, seja de fato uma porta de entrada para o SUS e realize a integralidade em toda a sua RAS. 


\section{REFERÊNCIAS}

1. Mendes EV. A construção social da atenção primária à saúde. Brasília: Conselho Nacional de Secretários de Saúde - CONASS, 2015.

2. Giovanella L, Mendonça MHM, Buss PM, Fleury S, Gadelha CAG, Galvão LAC, et al. De Alma-Ata a Astana. Atenção primária à saúde e sistemas universais de saúde: compromisso indissociável e direito humano fundamental. Cad Saúde Pública. 2019; 35 (3): 1-6.

3. Ministério da Saúde (BR). Secretaria de Atenção à Saúde. Departamento de Atenção Básica. Política Nacional de Atenção Básica. Brasília: Ministério da Saúde, 2012.

4. Brasil. Constituição (1988). Constituição da República Federativa do Brasil. Brasília, DF: Senado Federal: Centro Gráfico, 1988.

5. Ministério da Saúde (BR). Secretaria de Atenção à Saúde. Departamento de Atenção Básica. Manual Instrutivo do Programa Nacional de Melhoria do Acesso e da Qualidade da Atenção Básica- Terceiro Ciclo (2015-2017) - Brasília: Ministério da Saúde, 2017.

6. da Fonseca Soppa FB, dos Santos RP, Guerin C, Vieira CS, Toso BRG. Planejamento estratégico na resolução de barreiras de acesso da atenção primária à saúde: revisão narrativa. Varia Scientia-Ciências da Saúde. 2017; 3 (1): 75-85.

7. Silva LS, Viegas SMF, Nascimento LC, Menezes C, Martins JRT, Potrich T. Universalidade do acesso e acessibilidade no quotidiano da atenção primária: vivências de usuários do sus. R Enferm Cent O Min. 2020; 10: 1-9.

8. Ministério da Saúde (BR). Secretaria de Atenção em Saúde. Departamento de Atenção Básica. Manual do instrumento de avaliação da atenção primária à saúde: primary care assessment tool pcatool - Brasil. Brasília: Ministério da Saúde, 2010.

9. Azevedo ALM, Costa AM. A estreita porta de entrada do Sistema Único de Saúde (SUS): uma avaliação do acesso na Estratégia Saúde da Família. Interface (Botucatu). 2007; 14 (35): 797-810

10. Oliveira MAC, Pereira IC. Atributos essenciais da Atenção Primária e a Estratégia Saúde da Família. Rev. Brasileira de Enfermagem. 2013; 66 (Esp):58-164.

11. Pesquisa Nacional de Saúde (PNS). PNS: informações sobre domicílios, acesso e utilização dos serviços de saúde : Brasil, grandes regiões e unidades da federação. IBGE, Coordenação de Trabalho e Rendimento. - Rio de Janeiro : IBGE, 2019.

12. Costa JP, Jorge MSB, Vasconcelos MGF, Paula MLD, Bezerra IC. Resolubilidade do cuidado na atenção primaria: articulação multiprofissional e rede de serviços. Saúde debate. 2014; 38 (103): 733-43.

13. Almeida PF, Fausto MCR, Giovanella L. Fortalecimento da atenção primária à saúde: estratégia para potencializar a coordenação dos cuidados. Rev. Panam. Salud. Publica. 2011; 29(2):84-95. 
14. Alfradique ME, Bonolo PDF, Dourado I, Lima-Costa MF, Macinko J, Mendonça CS, et al. Internações por condições sensíveis à atenção primária: a construção da lista brasileira como ferramenta para medir o desempenho do sistema de saúde (Projeto ICSAP - Brasil). Cad. Saúde Pública. 2009; 25(6):1337-49.

15. Prodanov CC, Freitas CF. Metodologia do trabalho científico [recurso eletrônico]: métodos e técnicas da pesquisa e do trabalho acadêmico. 2. ed. - Novo Hamburgo: Feevale, 2013.

16. Castro RCLD, Knauth DR, Harzheim E, Hauser L, Duncan BB. Avaliação da qualidade da atenção primária pelos profissionais de saúde: comparação entre diferentes tipos de serviços. Cad. saúde pública. 2012; 38 (Esp): 279-95.

17. Reis RS, Coimbra LC, Silva AAMD, Santos AMD, Alves MTSSDB, Lamy ZC, et al. Acesso e utilização dos serviços na estratégia saúde da família na perspectiva dos gestores, profissionais e usuários. Ciênc. saúde coletiva. 2013; 18(11): 3321-31.

18. Lins ÍL, de Luna Neto RT, Leite SFP, de Oliveira JMC, Felipe FA, Ferreira NB. Avaliação da assistência prestada na estratégia saúde da família na ótica do usuário. Revista de Psicologia. 2014; 8 (24).

19. Silva SAD, Nogueira DA, Paraizo CMDS, Fracolli LA. Avaliação da Atenção Primária à Saúde: visão dos profissionais de saúde. Rev. Esc. Enferm. USP. 2014; 48 (Esp): 122-8.

20. Vitoria AM, Harzheim E, Takeda SM, Hauser L. Avaliação dos atributos da atenção primária à saúde em Chapecó, Brasil. Rev. Bras. Med. Fam. Comunidade. 2013; 8(29): 285-93.

21. Chomatas ERDV, Vigo A, Marty IK, Hauser L, Harzheim E. Avaliação da presença e extensão dos atributos da atenção primária em Curitiba. Rev. Bras. Med. Fam. Comunidade. 2013; 8 (29): 294-303.

22. César MDC, Campos GWDS, Montebelo MIDL, Sarmento G. Avaliação da atenção primária no município de Piracicaba, SP, Brasil. Rev. Saúde Debate. 2014; 38 (esp): 296-306.

23. Oliveira WN. O olhar da população idosa sobre o acesso a uma unidade básica de saúde em Porto Alegre- RS. Rev. Fac. Odontol. 2010; 51 (3): 17-22.

24. Silva DM, Nascimento MM, Tenorio DB. Desenvolvimento de um sistema web como facilitador de marcação de consultas no sistema público de saúde de Guarulhos para redução de filas presenciais. Rev. Computação Aplicada. 2020; 9 (1).

25. Sala A, Luppi CG, Simões O, Marsiglia RG. Integralidade e atenção primária à saúde: avaliação na perspectiva dos usuários de unidades de saúde do município de São Paulo. Saúde Soc. 2011; 20 (4): 948-60.

26. Oliveira BRG, Viera CS, Collet N, de Lima RAG. Acesso de primeiro contato na atenção primária em saúde para crianças. Rev Rene. 2012; 13(2): 332-42. 
27. Lemos SS,Spiri WC, Spagnuolo RS. A percepção dos enfermeiros da atenção básica sobre a integralidade na saúde. Rev. Eletrônica Acervo Saúde. 2020; 12(10): 1-8.

28. Cunha ABO, Vieira-da-Silva LM. Acessibilidade aos serviços de saúde em um município do Estado da Bahia, Brasil, em gestão plena do sistema. Cad. Saúde Pública. 2010; 26 (4): 725-37.

29. Medeiros FA, de Araújo-Souza GC, Albuquerque-Barbosa AA, Clara-Costa IDC. Acolhimento em uma Unidade Básica de Saúde: Satisfação do usuário em foco. Revista. Saulud Pública. 2010; $12(3): 402-13$.

30. Gomide MFS, Pinto IC, Bulgarelli AF, Santos ALPD, Serrano Gallardo MDP. A satisfação do usuário com a atenção primária à saúde: uma análise do acesso e acolhimento. Interface (Botucatu). 2017; 22(65): 387-98.

31. Ministério da Saúde (BR). Secretaria de Atenção à Saúde. Departamento de Atenção Básica. Política Nacional de Humanização - PNH. Brasília, 2013.

32. Camargo DS, Castanheira ERL. Ampliando o acesso: o Acolhimento por Equipe como estratégia de gestão da demanda na Atenção Primária à Saúde (APS). Interface (Botucatu). 2020; 24 (1): 1-17.

33. Mendes LRN, Chaves JC, Ramos MCA, Alves LD, Diamantino MLP, Bragança MRF et al. Integralidade da atenção à saúde e integração dos serviços em uma unidade básica de atenção primária a saúde de Governador Valadares, MG, 2019. APHAC, (6): 44-51.

34. Siochetta TM, da Silva A, Beuren AC, de Almeida SDM. Baixa resolutividade na rede de atenção à saúde: um problema vigente. Revista saúde integrada. 2019; 12 (23): 190-203.

35. Azevedo ALDCS, Pereira AP, Lemos C, Coelho MF, Chaves LDP. Organização de serviços de emergência hospitalar: uma revisão integrativa de pesquisas. Rev. Eletr. Enferm. 2010; 12 (4):736-45.

36. Rodrigues MM, Alvarez AM, Rauch KC. Tendência das internações e da mortalidade de idosos por condições sensíveis à atenção primária. Rev Bras Epidemiol. 2019; 22: 1-11.

37. Maia LG, Silva LA, Guimarães RA, Pelazza BB, Pereira ACS, Rezende WL et al. Hospitalizations due to primary care sensitive conditions. Rev Saúde Pública. 2018; 53: 1-11.

38. Nied MM, Bulgarelli PT, Rech RS, Buno CS, Santos CM, Bulgarelli AF. Elementos da Atenção Primária para compreender o acesso aos serviços do SUS diante do autorrelato do usuário. Cad saúde colet. 2020; 28 (3): 362-72.

39. Silva ACS, Soares IRB, Campos KFC, Castro MCS. A Atenção Primária sob a ótica dos usuários do Sistema Único de Saúde: uma revisão bibliográfica. Rev SUSTINERE. 2019; 7 (1): 46-72.

40. Vázquez ML, Vargas I, Unger JP, Mogollón A, Silva MRFD, Paepe PD. Integrated health care networks in Latin America: toward a conceptual framework for analysis. Rev. panam. salud pública. 2009; 26: 360-7. 
41. Silva TRS, Santos JCM, Oliveira JS, Abreu VPL, Silva RR, Dantas KL, et al. A importância do rastreamento do câncer do colo do útero e fatores relacionados à não adesão.Res., Soc. Dev. 2021; 10(4): 2-12

\section{DADOS DOS AUTORES}

\section{Rita Oliveira Costa}

Residente da Fundação Escola De Saúde Pública De Palmas (FESP), Palmas/TO - Brasil. ritacostao@gmail.com

\section{Maitê da Veiga Feitoza Borges Silva}

Enfermeira da Capitalent Medical, Alemanha. maite.vfbs@gmail.com

\section{Carolina Freitas do Carmo Rodrigues}

Enfermeira Epidemiologista do Instituto Brasileiro De Desenvolvimento Da Administração Hospitalar (IBDAH), Salvador/BA - Brasil. carolppgecs@gmail.com

\section{Ruhena Kelber Abrão Ferreira}

Professor do Magistério superior na Universidade Federal Do Tocantins (UFT), Palmas/TO-Brasil. kelberabrao@uft.edu.br

\section{Cristina Vianna Moreira dos Santos}

Professora do Magistério superior na Universidade Federal do Tocantins (UFT), Palmas/TO - Brasil.cristinavianna@uft.edu.br

Submetido em: 6-6-2021

Aceito em: 28-7-2021 